\title{
Open Surgical Repair of Abdominal Aortic Aneurysms Maintains a Pivotal Role in the Endovascular Era
}

\author{
Christopher D. Blackstock, MD, PhD ${ }^{1}$ Benjamin M. Jackson, MD, FACS ${ }^{1}$ \\ ${ }^{1}$ Division of Vascular Surgery and Endovascular Therapy, University of \\ Pennsylvania, Philadelphia, Pennsylvania \\ Semin Intervent Radiol 2020;37:346-355 \\ Address for correspondence Christopher D. Blackstock, MD, PhD, \\ Division of Vascular Surgery and Endovascular Therapy, Hospital of the \\ University of Pennsylvania, 3400 Spruce Street, Maloney-4, \\ Philadelphia, PA 19104 \\ (e-mail: christopher.blackstock@pennmedicine.upenn.edu).
}

\author{
Abstract \\ Keywords \\ - abdominal aortic \\ aneurysm \\ - open aortic surgery \\ - aortic repair \\ - endovascular aortic \\ repair \\ - interventional \\ radiology
}

\begin{abstract}
Since the advent of endovascular aortic repair (EVAR) nearly three decades ago, there has been a paradigm shift in the treatment of the abdominal aortic aneurysm (AAA) to favor EVAR due to its reduced operative mortality, less invasive nature, and faster recovery times. However, more recently there has been an accumulation of data from large meta-analyses and randomized clinical trials revealing that EVAR has no survival benefit after approximately 2 years and is associated with substantially higher rates of reintervention and aneurysm rupture in the long term. These findings call into question the durability of EVAR compared with open aortic repair and emphasize the need for surgeons to remain competent with open aortic surgery in the modern era. This article will provide comprehensive review of a large body of literature comparing endovascular repair to open aortic surgery for the management of AAAs, and it will offer an overview of the open surgical repair technique for AAAs.
\end{abstract}

In 2020, the primary treatment options for the management of the abdominal aortic aneurysm (AAA) consists of either traditional open aortic surgery or endovascular exclusion. ${ }^{1}$ With the less invasive nature and early mortality benefit of endovascular aortic repair (EVAR) seen in major randomized control trials, 2,3 endovascular strategies have gained tremendous popularity in the modern era while open surgery case numbers have dwindled. It is estimated that by 2010 , EVAR accounted for $78 \%$ of all existing AAA repairs. ${ }^{4}$ Concurrently, the total open AAA repairs in Medicare beneficiaries declined by $76 \%$ over one decade. ${ }^{5}$ Currently, approximately $80 \%$ of all AAAs are treated with EVAR, and there is expected further growth of the endovascular strategy as devices and capabilities continue to advance. ${ }^{6}$ However, there are still many limitations of EVAR, as AAAs often present with a variety of complexities and may have challenging features that prohibit repair with standard EVAR. Therefore, open aortic repair continues to fulfill a fundamental role in the modern era.

\section{Open Surgical Repair versus Endovascular Aortic Repair}

The reduced operative mortality, shorter length of stay (LOS), and faster recovery times with EVAR have made it an attractive option, especially in patients with severe comorbidities. However, the long-term trajectory and outcomes after EVAR and how it compares to traditional open repair are still being elucidated. Four major prospective randomized clinical trials (RCTs) have compared EVAR to open surgical repair for large infrarenal AAAs and looked at outcomes on survival and complications requiring reinterventions. -Fig. 1 summarizes some of the major endpoints for each trial. ${ }^{2,3,7,8}$ Importantly, patients were included for the RCTs only if they met criteria to be candidates for both open aortic repair and EVAR. Overall, the data consistently show that although EVAR offers reduced 30-day mortality, this survival benefit is not sustained by midterm; and EVAR is associated with a substantially higher rate of reinterventions. The two largest
Issue Theme Aortic Interventions; Guest Editors, Benjamin M. Jackson, MD, FACS and S. William Stavropolous, MD, FSIR
Copyright $\odot 2020$ by Thieme Medical Publishers, Inc., 333 Seventh Avenue, New York, NY 10001, USA. Tel: +1(212) 760-0888. 


\begin{tabular}{|l|c|c|c|c|c|c|c|c|c|}
\hline \multirow{2}{*}{$\begin{array}{l}\text { Randomized } \\
\text { Control Trial }\end{array}$} & \multicolumn{3}{|c|}{ 30-day Mortality (\%) } & \multicolumn{3}{c|}{ Overall Mortality (\%) } & \multicolumn{3}{c|}{ Secondary Intervention (\%) } \\
\cline { 2 - 11 } & OAR & EVAR & $p$ value & OAR & EVAR & $p$ value & OAR & EVAR & $p$ value \\
\hline EVAR-1 & 4.3 & 1.8 & 0.02 & 71 & 74 & 0.14 & 12 & 26 & $<0.0001$ \\
\hline DREAM* & 4.6 & 1.2 & 0.1 & 30.1 & 31.1 & NS & 18.1 & 29.6 & $<0.05$ \\
\hline OVER & 3 & 0.5 & 0.004 & 70 & 68 & 0.61 & 19.8 & 26.7 & $<0.05$ \\
\hline ACE & 0.6 & 1.3 & NS & 8 & 11.3 & 0.24 & 2.7 & 16 & $<0.0001$ \\
\hline
\end{tabular}

Fig. 1 Compilation of major endpoints from the major randomized clinical trials comparing endovascular aneurysm repair (EVAR) to open aortic surgery (OAR) for the treatment of abdominal aortic aneurysms. Values are reported as percentage (\%) of total patients in each group. "NS" indicates no statistical significance, $p>0.05$. *DREAM overall mortality and secondary interventions are listed as 100 minus the reported \% survival or \% freedom from secondary intervention. EVAR-1, ${ }^{2}$ United Kingdom Endovascular Aneurysm Repair trial 1; follow-up: 1.8-15.8 years, median 12.4 years. DREAM, ${ }^{7}$ Dutch Randomized Endovascular Aneurysm Management trial; follow-up: 5-7 years, reported at 6 years. OVER, ${ }^{3}$ Open versus Endovascular Repair trial; follow-up: $0.02-14.2$ years, median 9.4 years. ACE, ${ }^{8}$ Anevrysme de l'aorte abdominale: Chirurgie versus Endoprothese trial; follow-up: $0-4.8$ years, median 3 years.

RCTs, the Endovascular Aneurysm Repair trial 1 (EVAR-1) and the Open Versus Endovascular Repair (OVER) trial, which included long-term follow-up up to 14 and 15 years postrepair, respectively, found conflicting results about overall survival after 8 years from repair. However, both of these trials, along with multiple large meta-analyses, identified a strong signal of late endograft failure and rupture with EVAR. $^{2,3}$ - Fig. $\mathbf{2}$ is adapted from a recent systemic review and meta-analysis of a large body of patient data from numerous studies, which consistently observed that the odds of secondary aortic rupture is nearly four- to fivefold more likely with EVAR compared with open aortic repair. ${ }^{9}$

EVAR-1 was the first and largest of the RCTs comparing open aortic repair to EVAR. It was performed in the United Kingdom and randomized 1,252 total patients. Thirty-day operative mortality was $1.8 \%$ in the EVAR group and $4.3 \%$ in the openrepair group $(p=0.02)$. However, the survival benefit of EVAR was lost by 1 to 2 years. In fact, after 8 years, the EVAR group had significantly higher all-cause mortality and aneurysmrelated mortality ( 7 vs. $1 \%$ ) than the open repair group, mainly attributable to secondary aneurysm sac rupture. Of note, the number of cancer-related deaths (excluding lung cancer) in the EVAR group after 8 years was also significantly higher than in the open repair group (adjusted hazard ratio [HR]: 1.87, 95\% confidence interval [CI]: 1.19-2.96; $p=0.007$ ), raising concern about the effects of long-term radiation associated with the more frequent surveillance imaging required with EVAR. However, this observation was not seen in the other trials and overall cancer-related deaths were not different between groups. Finally, the numbers of life-threatening, serious, and total reinterventions were all significantly higher (more than twofold) in the EVAR group. ${ }^{2}$

The OVER trial was performed in the United States at multiple Veterans Affairs medical centers and randomized 881 veterans with AAAs, including 41 patients with AAA $<5.0 \mathrm{~cm}$ that had an associated iliac artery aneurysm $\geq 3.0 \mathrm{~cm}$, rapid enlargement, or saccular morphology. Similar to EVAR- 1 and other RCTs, OVER observed an early mortality benefit with EVAR (0.5 vs. $3.0 \% ; p=0.004)$, which was not sustained at 2 years, and overall mortality was no different between groups. In contrast to EVAR-1, mortality after 8 years was not statistically higher with EVAR. However, like EVAR-1,

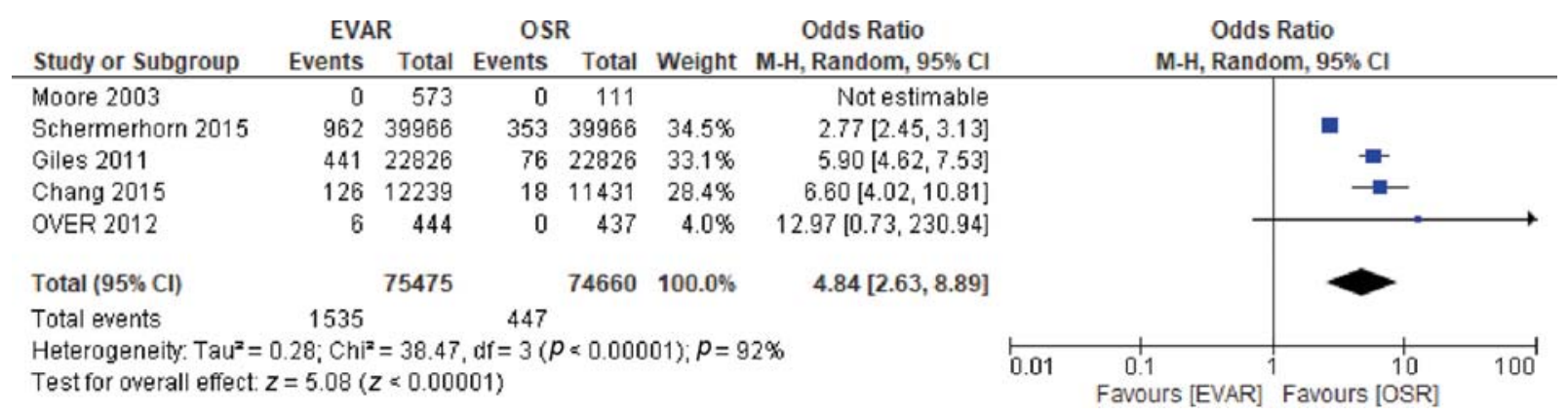

Fig. 2 Meta-analysis of endovascular aneurysm repair (EVAR) vs. open surgical repair (OSR) long-term secondary rupture rate including studies with 5 to 9 years of follow-up. (Moore 2003 was excluded because there was an event rate of $0 \%$ in both groups, leading to a nonestimable odds ratio $[\mathrm{OR}])$. The solid squares are proportional to the weights used in the meta-analysis. The solid vertical line indicates no effect. The horizontal lines represent the $95 \%$ confidence interval $(\mathrm{CI})$. The diamond indicates the weighted odds ratio, and the lateral tips of the diamond indicate the associated 95\% CI. M-H, Mantel-Haenszel; OVER, Open versus Endovascular Repair Veterans Affairs Cooperative Study; Random, random effects. (Data from Li B, Khan S, Salata K, et al. A systematic review and meta-analysis of the long-term outcomes of endovascular versus open repair of abdominal aortic aneurysm. J Vasc Surg 2019;70(3):954-969.e30.) 
aortic rupture occurred significantly more frequently in the EVAR group, (seven patients in EVAR group vs. one patient in open aortic repair group). The one rupture in the open repair group occurred in the thoracic aorta. ${ }^{3}$ Of a side note, there were no significant differences in erectile dysfunction (a secondary outcome of the study) at 3, 6, and 12 months, despite this complication thought to be more prevalent with open repair. ${ }^{10}$

While these RCTs, by definition, provide the least biased results comparing EVAR to open surgery, these studies have limitations. They have been criticized for "poor generalizability" in representing the AAA population as a whole, as these studies only included patients with infrarenal AAAs who meet criteria for both open surgery and standard EVAR. $75 \%$ of the patients with AAAs initially screened in EVAR-1 and $52 \%$ of those screened for OVER were excluded. Furthermore, the inclusion criteria may have selected for a patient population favorable for EVAR. For instance, in EVAR-1, 54\% of patients screened were eliminated due to unsuitable EVAR anatomy, and the average aortic neck length of patients in the RCTs was longer than what is seen in the routine practice setting. ${ }^{11}$ It is well established that a longer proximal seal zone correlates with reduction in the rate of endograft migration, proximal endoleak, and subsequent aneurysm sac expansion. ${ }^{1}$ Thus, it is conceivable that the selective patient population in the RCTs with favorable anatomy for EVAR may have led to better outcomes than what would have been observed in actual practice. In reality, EVAR is frequently used outside of the device instructions for use (IFU). In a separate study using the M2S, Inc. imaging database from 1999 to 2008 , Schanzer et al found that only $42 \%$ of patients treated with EVAR had anatomy that met the most conservative definition of the device IFUs, including an aortic neck seal zone of $1.5 \mathrm{~cm} .^{12}$

Given the limitations of the RCTs and their somewhat conflicting findings on long-term survival, many large metaanalyses have been performed in effort to gain clarity. Schermerhorn et al performed a retrospective propensityscore-matched cohort study containing 39,966 matched pairs of Medicare beneficiaries who underwent AAA repair between 2001 and $2008 .{ }^{4}$ In contrast to the RCTs, the decision for the type of repair (open vs. EVAR) was determined by the surgeon and patients may not have been candidates for both types of repair. In this highly powered analysis, perioperative mortality (5.2\% with open repair vs. $1.6 \%$ with EVAR) was very similar to what was observed in RCTs, and again this mortality benefit was lost by midterm follow-up (3 years), and there was no overall survival difference at 8 years. Also consistent with RCTs, EVAR had a significantly higher reintervention rate than open repair. However, this difference was smaller than what had been observed in EVAR-1 because this analysis included all laparotomy-related complications, which accounted for the majority of late reinterventions in the open repair group. EVAR had significantly higher rates of overall, minor, and major complications requiring reintervention, including persistent endoleak, stent migration, thrombosis, and aortic rupture. Strikingly, the rate of aneurysm-related reinterventions was markedly higher with EVAR (18.8\% compared with
$3.7 \%$ with open repair, $p<0.001$ ), and the rate of AAA rupture was substantially higher with EVAR compared with open repair ( 5.4 vs. $1.4 \%, p<0.001$ ). These findings are particularly concerning and raise question about the durability of endovascular repair compared with traditional open surgery. 4

\section{Aortic Rupture}

Aortic rupture is the catastrophic ultimate manifestation of AAA disease, which results in massive hemorrhage and death for most patients. Of all aortic ruptures that occur in the community setting, approximately $50 \%$ of patients die before they make it to the hospital. Of those who make it to the hospital, only about half survive to be discharged. Large community-based studies reported mortality rates of 85 to $90 \%$ with AAA rupture. ${ }^{13}$ Given the less invasive nature of EVAR and low operative mortality seen in the repair of asymptomatic AAA patients, there has been hope that EVAR could lessen this dismal sequela of ruptured AAA. However, none of the major RCTs comparing open to endovascular repair of ruptured AAA have shown a difference in early mortality between approaches. ${ }^{14-16}$

The first two of these major RCTs were the ECAR trial (Endovasculaire versus Chirurgie dans les Anévrysmes Rompus) ${ }^{14}$ in France and the AJAX trial (Amsterdam Acute Aneurysm $)^{15}$ in the Netherlands. Patients were included only if their anatomy was suitable for EVAR, and primary endpoints included 30-day mortality. These studies had relatively small sample sizes (116 and 107, respectively), which had been powered based on a robust survival benefit of EVAR that was previously seen in many observational studies. However, these RCTs failed to show survival benefit with EVAR. The largest and most recent trial was the IMPROVE trial (Immediate Management of the Patient with Ruptured Aneurysm: Open Versus Endovascular repair $)^{16}$ in the United Kingdom and Canada. It was higher powered with 613 patients, and the randomization design differed to include patients with anatomy unfit for EVAR. At the point of clinical diagnosis, patients were randomized to EVAR if found anatomically suitable on CT, versus open repair if not. Even with this design that presumably selected those with less challenging anatomy for EVAR, IMPROVE failed to show a statistical difference in mortality at 30 days (35\% with EVAR vs. $37 \%$ with open) and at 1 year (all-cause mortality was $41.1 \%$ for the EVAR group vs. $45.1 \%$ for the open repair group, $p=0.325) .^{17}$

In stark contrast to these RCTs, multiple large observation studies, including a propensity-matched cohort study using the VQI database and in Medicare beneficiaries, show a substantial survival benefit of EVAR compared with open repair for ruptured AAAs. ${ }^{18,19}$ A recent study matched cohort using real-world evidence from the Premier Healthcare Database between 2009 and 2015, showed significantly higher adjusted mortality with open aortic repair compared with EVAR ( 36.3 vs. $23.8 \% ; p<0.001$ ) as well as a higher complication rate. ${ }^{20}$ Importantly, mortality was adjusted for differences in demographics, hospital characteristics, and comorbidities, 
but it did not take into account anatomic constraints. Thus, it is conceivable that those elected for open repair had more challenging anatomy, which could account for worse outcomes in those patients. Hostile aortic anatomy can lead to poor outcomes and increased mortality with endovascular repair, but has not been shown to affect outcomes with open repair. ${ }^{21}$ Thus, while potential survival benefit of EVAR for ruptured AAA remains to be elucidated, open aortic repair is certainly the mainstay treatment for many patients whose anatomy is unfavorable to EVAR.

\section{Durability of Endovascular Aortic Repair}

Although endovascular therapies may be a reasonable option for patients who cannot tolerate an extensive open aortic repair, multiple studies have raised concern about the longterm durability of EVAR. Schanzer et al reported that the 5-year post-EVAR rate of AAA sac enlargement was $41 \%{ }^{12}$ Furthermore, sac expansion significantly correlates with late mortality. At 5-year post-EVAR, survival in patients with AAA sac expansion ( $>5 \mathrm{~mm}$ ) was $68 \%$ compared with $83 \%$ in patients without sac expansion $(p<0.001) .^{22}$ Endoleak is an independent predictor of sac expansion and can be considered the "Achilles' heel" of EVAR. In fact, evidence suggests that endoleak is the predominant cause of late ruptures after EVAR. ${ }^{23}$-Fig. 3 shows angiography demonstrating a type la endoleak (a), type la endoleak with rupture (b), and type III endoleak with rupture (c).

Several studies have suggested that EVAR performed outside of device-specific IFUs may contribute to endoleak. In a large multicenter retrospective study in Canada, $43.8 \%$ of patients underwent EVAR with violation of device-specific IFU, most frequently in regard to neck diameter and angulation. Furthermore, violation in IFUs was significantly associated with adverse outcomes including endoleak and aneurysm-related mortality. ${ }^{24}$ We know that after treatment with EVAR, there is often proximal neck dilation that occurs overtime, even when there is early AAA sac regression. Although a proximal seal may be achieved initially, neck angulation or inadequate seal length may increase tendency for neck dilation and susceptibility to development of type Ia endoleak. ${ }^{25}$ This notion was further supported by a retrospective case-control study evaluating endovascular techniques performed on AAAs with angulated necks outside of IFU and found increased development of type Ia endoleak in the long term (i.e., 7 years). These endoleaks had not been present at early or midterm, but were associated with proximal neck enlargement over time. ${ }^{26}$ These studies highlight the importance of achieving adequate proximal seal zone to prevent the development of type 1a endoleak and risk of subsequent rupture.

\section{The Technique of Open Aortic Surgery}

The technique of open surgery for AAA repair as it is performed today was first described in the 1950s; however, records of the description of aneurysms and their treatment date back to as early as the Egyptians around 2000 BC. In 1951, Charles Dubost in Paris performed the first successful resection of an AAA with graft replacement. ${ }^{27}$ Shortly after this, Denton Cooley described performing the first repair of a ruptured AAA with homograft. ${ }^{28}$ Also around this time, Michael DeBakey created the Dacron graft for the replacement of the aneurysmal aorta, which is essentially the standard arterial graft used for AAA repair in practice today. ${ }^{29}$ Graft configurations vary depending on aneurysm extension. A straight tube graft is preferred when suitable, and is sewn end to end to the aorta, proximally and distally. A bifurcated graft is used in the setting of aneurysmal disease involving the distal aorta or iliac vessels and can be sewn distally to the iliac or femoral arteries. A graft with a proximal side arm can be used to reimplant a renal or visceral artery.

Open aortic repair can be performed from either a retroperitoneal or transabdominal approach. The transabdominal approach is customarily performed by making a midline abdominal incision extending from xiphoid to symphysis pubis. The ligament of Treitz is divided and retroperitoneum is incised to the left of the aorta. For an infrarenal clamp, the retroperitoneum is incised to expose the aorta up to the level of left renal vein. Mobilization or division of the left renal vein to facilitate suprarenal aortic clamping is often necessary. Distal clamping is often done at the level of the iliac arteries because aneurysmal disease frequently extends to
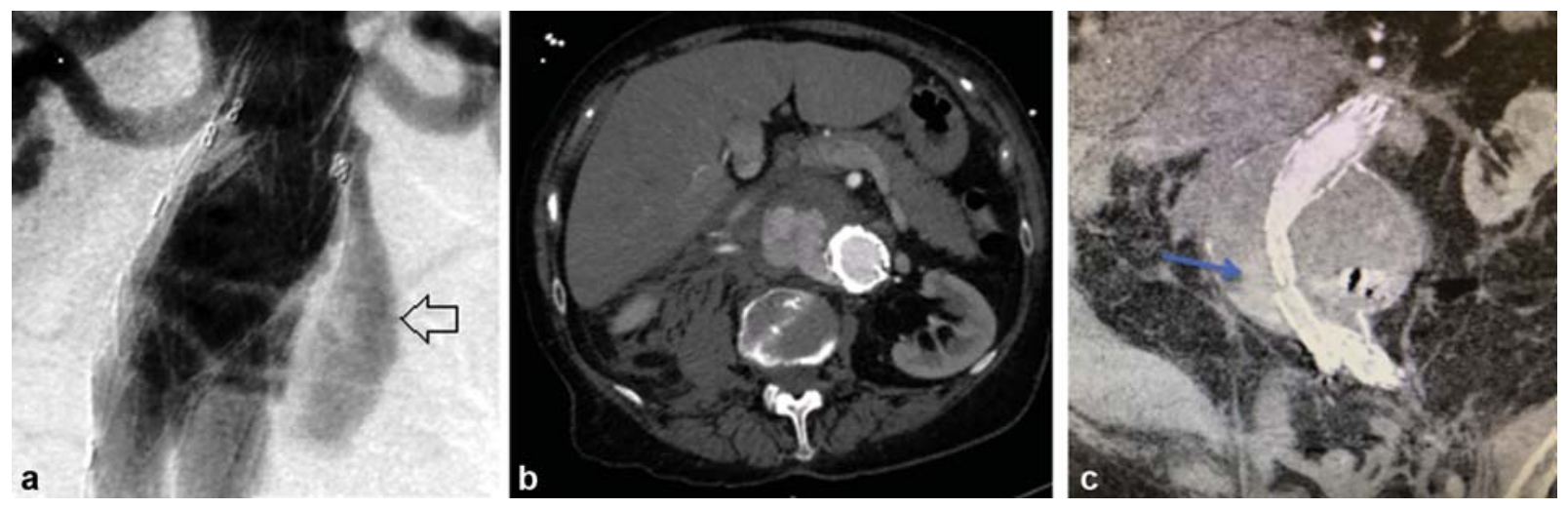

Fig. 3 (a) Angiography demonstrating type la endoleak (arrow). (b) Computed tomography angiography axial image showing type la endoleak with aortic rupture. (c) Computed tomography angiography coronal image showing type III endoleak from iliac limb component separation (arrow), causing aortic rupture. 
the aortic bifurcation, even when using a tube graft sewing to the distal aorta. ${ }^{6}$ When exposing the iliac vessels, meticulous care should be taken to avoid division of the nerves erigentes, which may result in erectile dysfunction or retrograde ejaculation.

For the retroperitoneal approach, the patient is positioned in the right lateral decubitus position, and the table is jackknifed to widen the space between the left costal margin and the left anterior superior iliac spine. An incision is typically made from the left 10 th intercostal space extended medially to just medial to the anterior iliac spine. The incision is carried through the flank musculature. The left kidney is mobilized anteriorly, and the left ureter is identified and is retracted anteriorly with the kidney. The left crus of the diaphragm must be divided to access the visceral aorta. - Fig. 4 demonstrates the sequential steps of the retroperitoneal approach for an infrarenal AAA repair using a bifurcated tube graft sewn distally to the bilateral common iliac arteries.

Usually, systemic heparin (80-100 units $/ \mathrm{kg}$ ) is administered prior to clamping the aorta, regardless of the approach. Typically, the sequence of clamping begins with the distal sites first followed by the proximal aorta to reduce the risk of distal embolization.

\section{Operative Risks and Complications}

Open aortic surgery is a major undertaking that is accompanied by notable operative risks. The overall operative mortality for open AAA repair historically averages around 5\%, as seen in the RCTs. However, lower mortality rates have been reported at high-volume institutions with high-volume surgeons. ${ }^{30}$ The majority of deaths in patients undergoing AAA repair are related to pulmonary or cardiac complications, and a large percentage of patients with AAAs also have chronic obstructive pulmonary disease (COPD) and/or coronary artery disease (CAD). A large meta-analysis found the occurrence of perioperative myocardial infarction and pneumonia with open AAA repair to be around 5 and 13\%, respectively. ${ }^{4}$ Despite preoperative optimization efforts, postoperative pneumonia and myocardial infarction after open aortic repair are not infrequent events and are commonly culprits in the operative mortality. ${ }^{4,6,31}$ Preoperative renal failure is also a predictor of mortality in patients undergoing AAA repair with both open surgery and EVAR. Postoperative renal dysfunction is a common complication after open AAA repair occurring in approximately $20 \%$ of patients, having an increased risk with a suprarenal aortic cross-clamping site. Some rare but dreaded complications that are uniquely associated with open repair compared with EVAR include anastomotic pseudoaneurysm formation, aortoenteric fistula, and sexual dysfunction or retrograde ejaculation. More commonly, laparotomy-related complications such as incisional hernia, bowel obstruction, and ileus can occur, particularly when using a transabdominal approach.

Outcomes after repair of ruptured AAA are worse compared with elective repair, regardless of repair approach. In a large single-center experience of open AAA repairs, the in-hospital mortality was $2.3 \%$ for elective cases versus $20.9 \%$ for urgent

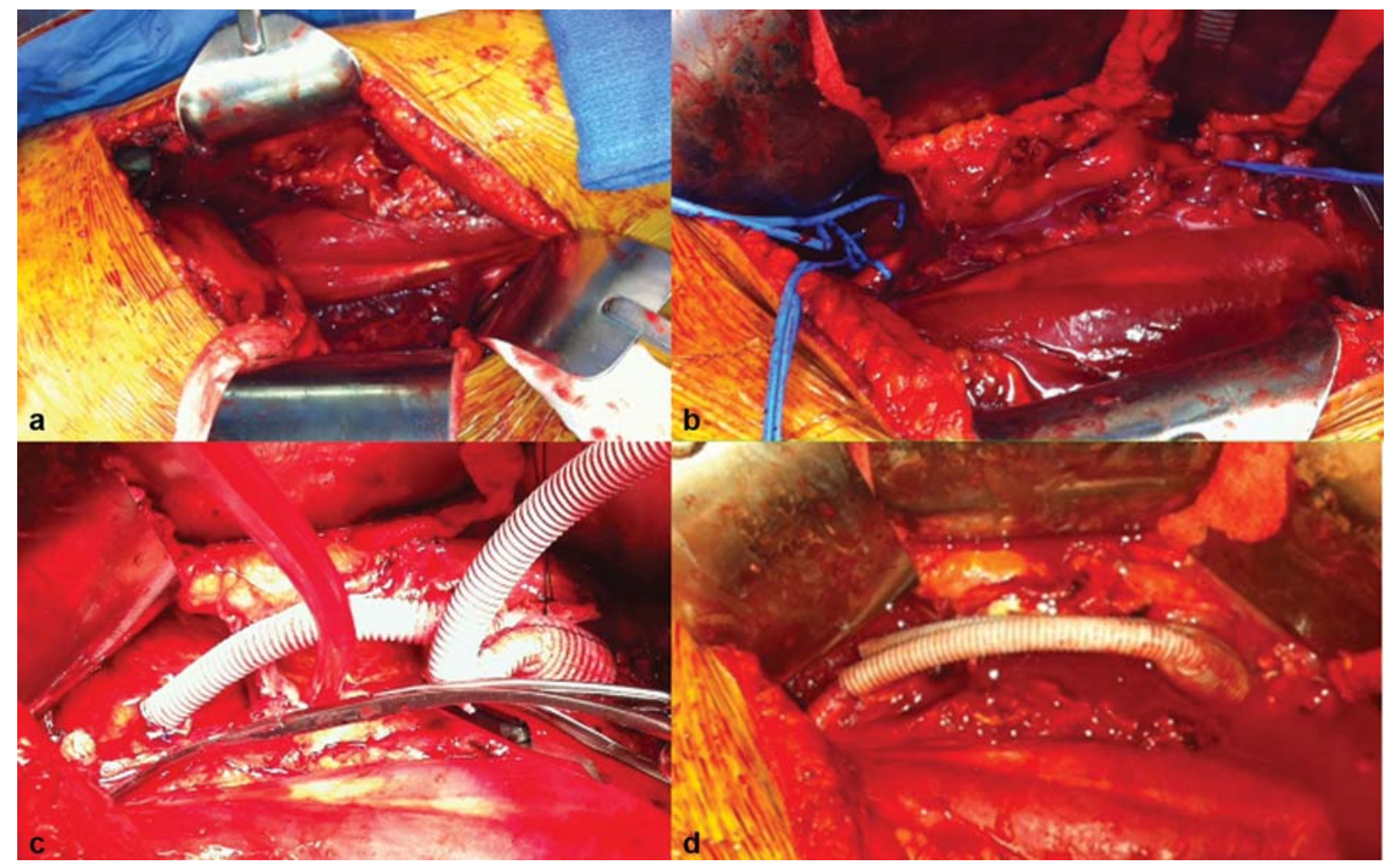

Fig. 4 Sequential steps for an infrarenal abdominal aortic aneurysm repair. (a) Left flank incision for retroperitoneal approach. (b) Vessel loop control of bilateral common iliac arteries and a retro-aortic left renal vein. (c) Dacron graft sewn to infrarenal aorta proximally and right limb sewn to right common iliac artery. Note vascular clamps on left iliac artery and left limb of aortic graft. (d) Completed aortic graft anastomoses to infrarenal aorta and bilateral common iliac arteries. Clamps removed to allow for reperfusion to lower extremities and pelvis. 
repair. ${ }^{30}$ Abdominal compartment syndrome is a major adverse sequela that can occur at a rate of approximately $7 \%$ after aortic rupture and repair with either EVAR or open surgery. Risk factors for developing abdominal compartment syndrome include use of aortic balloon occlusion, coagulopathy, massive transfusion, and use of an aorto-uni-iliac (AUI) stent graft. Ischemic colitis is another complication with increased risk after ruptured AAA repair, occurring in as many as 20 to $30 \%$ of patients. ${ }^{6}$

\section{Aortic Cross-Clamping and Complex AAAs}

AAA classification is based on proximal extension of the aneurysm to the level of the major visceral branches, as depicted in - Fig. 5. ${ }^{1}$ The extent of aneurysmal involvement at the visceral segment determines the device selection and case complexity for EVAR, or the clamp and sew sites for open aortic repair. Identifying an aortic cross-clamp site is a critical consideration for open repair. The ideal clamp site should be a nondiseased segment of aorta as distal as possible to minimize or eliminate visceral and renal ischemia. Clamping of calcified atherosclerotic plaque may cause fracture of the aortic wall, which can result in excessive hemorrhage. Suprarenal clamping compared with infrarenal clamping is, not surprisingly, associated with higher postoperative renal insufficiency and a higher overall complication rate; however, similar mortality rates have been observed. ${ }^{32}$ When comparing clamping above one renal artery versus both renal arteries, a large cohort from the American College of Surgeons National Surgical Quality Improvement Program (NSQIP) showed no difference in mortality or postoperative renal insufficiency. However, in comparison to suprarenal clamp, supraceliac clamping was associated with higher mortality and higher major complication rate including new dialysis and unplanned reoperation. ${ }^{33}$ Others had also observed increased mortality and postoperative renal dysfunction with supraceliac clamping (compared with suprarenal clamping), despite shorter clamp times. ${ }^{34}$ These findings strongly support choosing a clamp site that avoids visceral ischemia when achievable.

Given the increased morbidity and mortality related to a more proximal aortic cross-clamp, there is hope that endovascular strategies may offer enhanced benefit in the repair of complex AAAs involving the visceral segment that would otherwise require a supraceliac clamp. However, endovascular repair options for juxtarenal and pararenal AAAs and extent IV TAAAs are limited. Only one endovascular device, the Cook

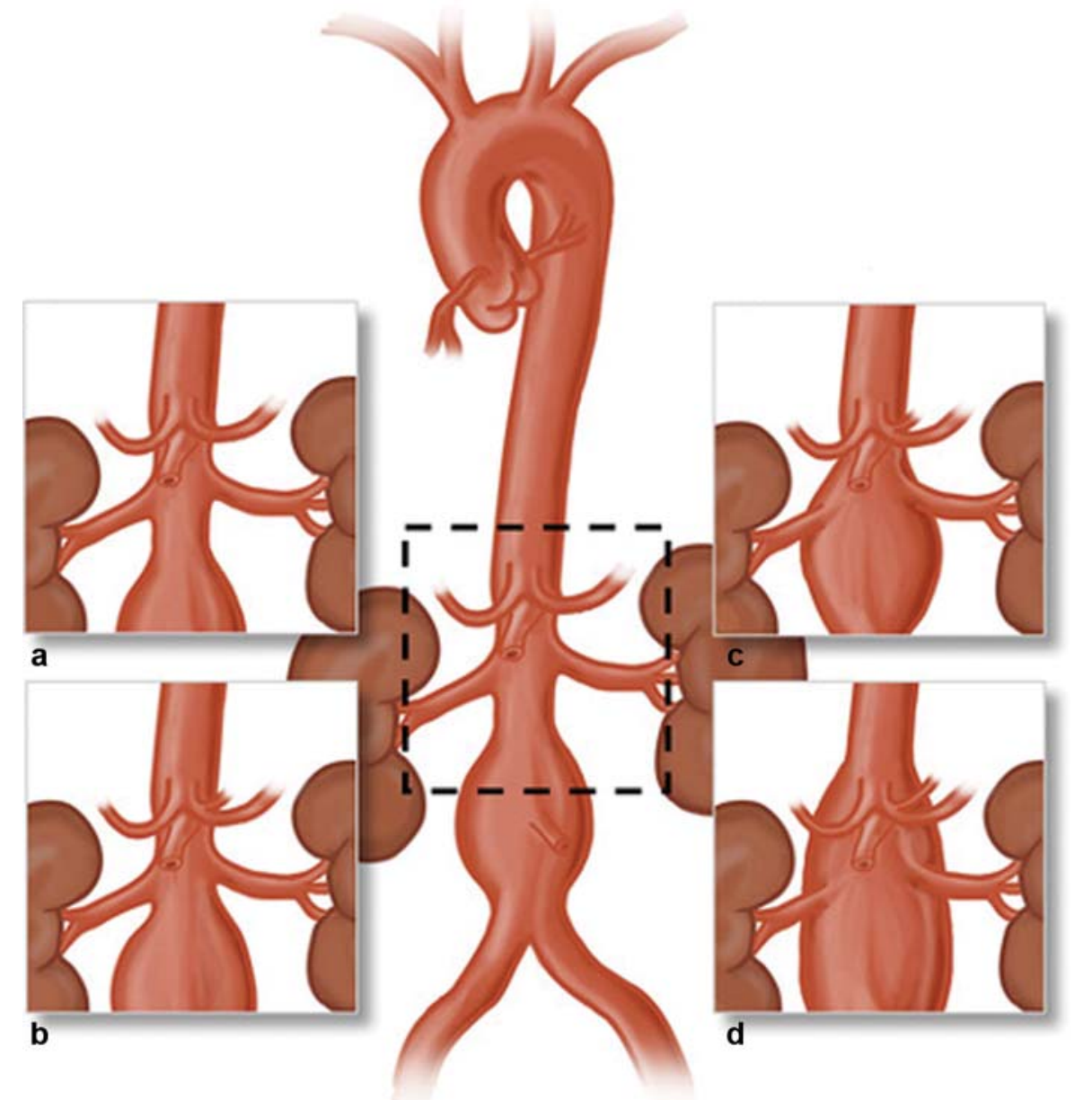

Fig. 5 Classification of proximal neck anatomy of abdominal aortic aneurysm (AAA). (a) Infrarenal AAA. (b) Juxtarenal AAA. (c) Suprarenal AAA. (d) Type IV thoracoabdominal aortic aneurysm. (Illustration by Winona W. Wu, MD, Division of Vascular and Endovasular Surgery, Beth Israel Deaconess Medical Center, Boston, MA. Reprinted with permission from Swerdlow NJ, Wu WW, Schermerhorn ML. Open and endovascular management of aortic aneurysms. Circ Res 2019;124(4):647-661.) 


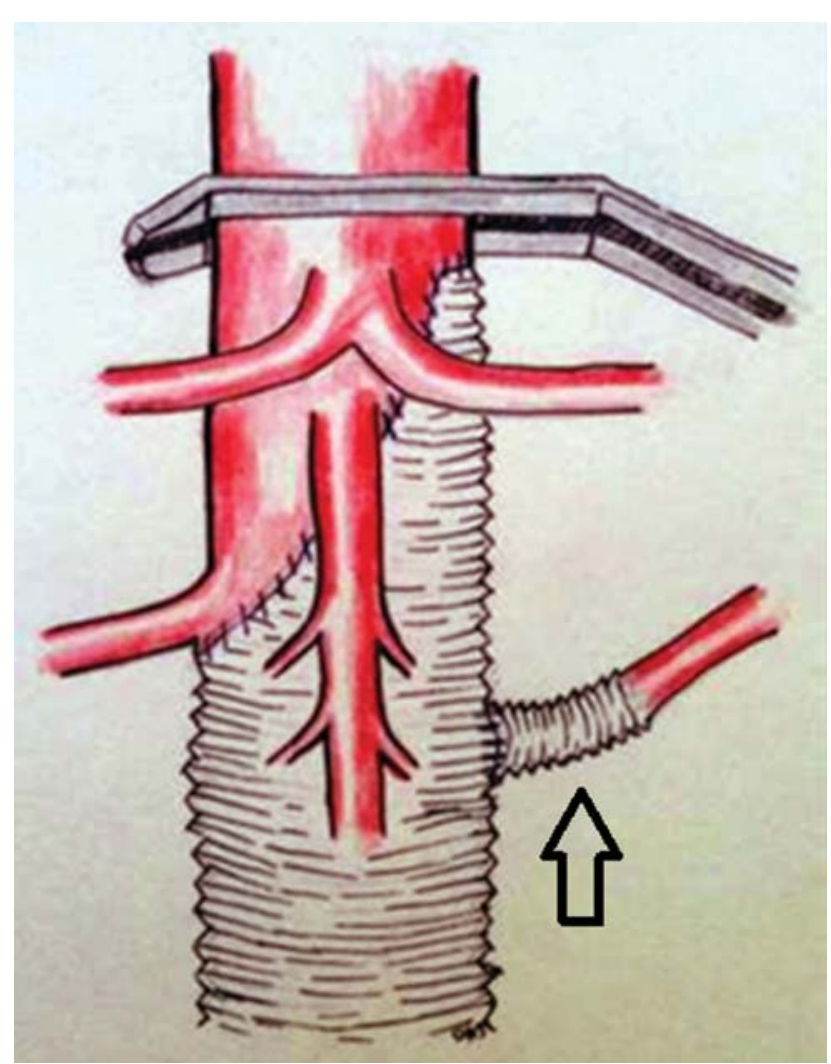

Fig. 6 Illustration of an open extent IV thoracoabdominal aortic aneurysm (TAAA) repair performed with a beveled proximal anastomosis incorporating the ostia of the celiac, superior mesenteric, and right renal arteries, using a Dacron graft with single side-arm for reimplantation of the left renal artery (arrow).

Zenith fenestrated endovascular graft (Z-fen), is approved for juxtarenal AAA repair. The industry-sponsored prospective multicenter trial, designed to evaluate the safety and effectiveness of the Z-fen, showed encouraging outcomes. In 67 patients, Oderich et al achieved 100\% technical success, 30-day mortality was $1.5 \%$ (one patient died of bowel ischemia), and 5-year survival was $91 \%$. At 5 years, there were no aneurysm ruptures or conversions to open repair, one patient developed a type Ia endoleak, and $22 \%$ required secondary intervention (primarily for renal artery stenosis or stent occlusion). ${ }^{35}$ These outcomes were comparable to those seen in the RCTs for infrarenal EVAR. However, there is no Food and Drug Administration-approved device designed to treat pararenal AAAs or extent IV TAAAs; therefore, open repair is the default approach in these situations, as illustrated in -Fig. 6.

\section{Obstacles to EVAR Favoring Open Repair}

One of the major obstacles to EVAR is aorto-iliofemoral occlusive disease, which may be prohibitive or increase the complexity and risks of adverse outcomes, including iliac artery rupture. It has been shown that among patients with AAA, 43\% have iliofemoral stenosis or occlusion. Traditionally, an iliac artery diameter of $7 \mathrm{~mm}$ is required for insertion of the main body device for EVAR. In a large single-institutional experience of over 400 patients undergoing EVAR at a tertiary academic medical center between 2009 and 2013, Etkin et al found that $48 \%$ of patients had difficulty of access (defined as having a diameter of iliac arteries smaller than $7 \mathrm{~mm}$ bilaterally). With the use of adjunctive techniques, including arterial dilators, iliac artery balloon angioplasty and stenting, AUI with femoral-femoral bypass, SoloPath balloon-expandable transfemoral access system, and direct iliac artery access via retroperitoneal flank incision, successful EVAR was made possible in the setting of iliac occlusive disease without change in complication rate. ${ }^{36}$ Additionally, newer and lower profile endovascular stent grafts have been designed specifically to accommodate narrow aortic bifurcations and iliac occlusive disease. Although devices and techniques are becoming more sophisticated, access site complications with EVAR are not infrequent, and occlusive disease may necessitate open aortic repair.

Likewise, concomitant iliac artery aneurysms will influence options for AAA repair. With regard to open repair, the transperitoneal approach is generally favored in the setting of a right common iliac artery aneurysm, because access to the right external and internal iliac arteries is limited from a left retroperitoneal approach. Endovascular exclusion of iliac artery aneurysms is an alternative approach, which may reduce the risks and morbidity associated with open repair. For concomitant common iliac artery (CIA) aneurysms in the setting of AAA, the EVAR limbs are often extended into the external iliac arteries to achieve an adequate distal seal zone. However, this usually requires embolization of the ipsilateral internal iliac artery to prevent a type II endoleak. Open repair of aortoiliac aneurysm was for long the favored approach, owing to the requirement to sacrifice one or both internal iliac arteries with EVAR. Recently, new endovascular devices have been designed particularly to treat iliac aneurysmal disease and preserve flow to both internal and external iliac arteries. The Gore Excluder Iliac Branch Endoprosthesis is commercially available, and the Cook Zenith Iliac Branch Device is under investigation.

\section{Special Circumstances: Connective Tissue Disorders}

While the majority of AAAs are considered to be of the degenerative phenotype that is closely associated with atherosclerotic disease, other etiologies of aortic aneurysm formation include infectious, inflammatory, and genetic. Genetically linked connective tissue disorders associated with aortic aneurysms include Marfan's syndrome (abnormal production of fibrillin-1 protein), vascular Ehlers-Danlos syndrome (defect in type III collagen synthesis), and Loeys-Dietz syndrome (mutation in transforming growth factor- $\beta$ receptor gene). Each of these syndromes have more aggressive rates of aneurysm expansion than degenerative aneurysms and are more likely to require intervention at an earlier age. ${ }^{37}$ Endovascular repair is not thought to be a primary option for treatment in these individuals due to poor outcomes, including high rates of endoleak and need for reintervention. ${ }^{38}$ In a relatively large single-center study with the use EVAR or TEVAR (thoracic endovascular aortic repair) for acute and chronic 
Marfan's aortic pathology, Waterman et al concluded that although endovascular therapy may provide an adjunct or bridge to open surgical treatment in select patients, failure of endovascular therapy in patients with Marfan's syndrome is common and associated with frequent need for reintervention. ${ }^{39}$ Thus, open aortic repair is the gold standard for the treatment of aortic pathology in Marfan's syndrome and other connective tissue disorders.

\section{Special Circumstances: Aortic Dissection}

Aneurysmal degeneration is a major adverse sequela of chronic aortic dissection that occurs from pathologic remodeling of the thoracic and abdominal aorta, which can be seen sporadically and in patients with connective tissue disorders. It is hypothesized that turbulent false lumen flow among other adverse hemodynamic changes results in loss of elastic lamina and deterioration of the media leading to aneurysmal degeneration. ${ }^{40}$ While treatment of aortic dissection with TEVAR can improve aortic remodeling in approximately two-thirds of patients, false lumen flow can persist and lead to late aneurysm formation. ${ }^{41}$ Aneurysmal degeneration occurs in approximately $40 \%$ of Stanford type B (aka DeBakey III) chronic dissections managed medically and in approximately onethird of patients treated with TEVAR. ${ }^{42}$ In a recent study in medically managed chronic DeBakey IIIB dissections, $83 \%$ of those with aneurysmal degeneration required aortic intervention. ${ }^{43}$ Often, aneurysm formation in chronic dissection affects the thoracic and abdominal aorta, requiring an extensive TAAA repair with relatively high rates of paraplegia. Staged procedures in which TEVAR is performed in adjunct with open extent III or extent IV TAAA repair have been described and may decrease paraplegic risk and enhance the technical feasibility of repair. ${ }^{44}$ Without adjunctive endovascular or open methods, sealing a stent graft in a dissected aorta is difficult or impossible, so while TEVAR is a mainstay of treatment for thoracic aortic dissections (in which the proximal seal is accomplished in normal undissected aorta, usually at the left subclavian artery takeoff), repair of the visceral and abdominal aorta and iliac arteries is most often accomplished with open repair when dissection results in aneurysmal degeneration.

\section{Special Circumstances: Need for Endograft Explantation}

Persistent endoleak with sac expansion warrants treatment. For type la endoleak, proximal extension with an aortic cuff is frequently used. However, proximal extension is not always effective or possible, and open surgery may be required. Besides in the setting of type la endoleak, failure of endovascular therapy is not an uncommon indication for open repair. Single-center retrospective studies have evaluated EVAR explanation and conversion to open aortic surgery performed for various indications including persistent endoleak (primarily type Ia), graft migration, thrombosis, rupture, and infection. A tube graft was used for repair in the majority of cases, and partial resection of the endograft was occasionally performed in select cases, with the distal anastomosis sewn to the distal unresected portion of the endograft. In the setting of an infected endograft, a rifampin-soaked or antibiotic-impregnated Dacron graft or aortic homograft can be used. For EVAR with suprarenal fixation devices, explantation can be especially challenging and may require a supraceliac aortic cross clamp. Overall, these experiences showed that while explantation of EVAR and conversion to open aortic repair is a major, technically challenging undertaking, it can be accomplished with morbidity and mortality rates similar to that of traditional open repair for complex AAAs. ${ }^{45,46}$

\section{Reevaluating the Treatment Paradigm}

The accumulation of data over recent years revealing the problems with EVAR durability has generated question of its widespread use as a primary treatment. With sac expansion rates reported over $40 \%$ at 5 years, substantially increased risk of rupture and reintervention rates compared with open surgery, as well as exceedingly high costs, it seems hard to justify the use of EVAR in patients who are candidates for open repair. In fact, the National Institute for Health and Care Excellence (NICE), a well-established and respected advisory institution in the United Kingdom, initially recommended against the use of EVAR completely in their 2018 "best practice" guidelines for the management of AAAs. This left open surgery as the only recommended intervention for asymptomatic AAAs, with patients who were not surgical candidates to be offered only conservative management with risk reduction. This created significant controversy with much of the vascular community and conflicted with the aims and endeavors of major organizations including the U.S. Society for Vascular Surgery and the European Society for Vascular Surgery, who were reluctant to abandon a rapidly advancing technique that seems to hold much potential. ${ }^{47}$ NICE revised their guidelines in March 2020, permitting the use of EVAR for asymptomatic infrarenal AAAs, but reserving its use to patients with comorbidities, anesthetic risks, or pathologies such as hostile abdomen that would make them poor candidates for open surgery. For complex AAA, the recommendations were less stringent and permitted offering EVAR to patients suitable to undergo both open surgical and endovascular repair, as long as candid discussion of the risks and uncertainties of EVAR was held with the patients, and they consented to enter the National Vascular Registry for research purposes. ${ }^{48}$ These modified recommendations seem reasonable and justified.

In conclusion, open aortic surgery remains the gold standard for AAA repair and should be considered in the majority of patients who can tolerate open surgery and have a life expectancy beyond a few years. While endovascular repair does have a place in the treatment of AAA for select patients, the aneurysm complication rate and risk of rupture seen with EVAR are particularly concerning. Patients for which EVAR may offer significant benefit over open aortic repair include those with short life expectancy ( $<2-5$ years) and those with significant comorbidities including COPD, CAD, congestive heart failure, or cerebrovascular disease which would pose high risk of the adverse outcomes with open repair. As endovascular techniques continue to advance and improve, its use in the treatment 
of complex AAAs that would otherwise require a supraceliac clamp and higher operative risk with open repair may be a particular area to benefit from endovascular therapy. However, for the majority of patients with infrarenal AAAs and certainly for those whose anatomy is not conducive to EVAR, the longterm data strongly support the continued utilization of open repair.

\section{Conflict of Interest}

None declared.

\section{References}

1 Swerdlow NJ, Wu WW, Schermerhorn ML. Open and endovascular management of aortic aneurysms. Circ Res 2019;124(04): 647-661

2 Patel R, Sweeting MJ, Powell JT, Greenhalgh RM, EVAR Trial Investigators. Endovascular versus open repair of abdominal aortic aneurysm in 15-years' follow-up of the UK endovascular aneurysm repair trial 1 (EVAR trial 1): a randomised controlled trial. Lancet 2016;388(10058):2366-2374

3 Lederle FA, Kyriakides TC, Stroupe KTOVER Veterans Affairs Cooperative Study Group, et al; Open versus endovascular repair of abdominal aortic aneurysm. N Engl J Med 2019;380(22):2126-2135

4 Schermerhorn ML, Buck DB, O'Malley AJ, et al. Long-term outcomes of abdominal aortic aneurysm in the Medicare population. N Engl J Med 2015;373(04):328-338

5 Suckow BD, Goodney PP, Columbo JA, et al. National trends in open surgical, endovascular, and branched-fenestrated endovascular aortic aneurysm repair in Medicare patients. J Vasc Surg 2018;67(06):1690-1697.e1

6 Chaikof EL, Dalman RL, Eskandari MK, et al. The Society for Vascular Surgery practice guidelines on the care of patients with an abdominal aortic aneurysm. J Vasc Surg 2018;67(01):2-77.e2

7 De Bruin JL, Baas AF, Buth JDREAM Study Group, et al; Long-term outcome of open or endovascular repair of abdominal aortic aneurysm. N Engl J Med 2010;362(20):1881-1889

8 Becquemin JP, Pillet JC, Lescalie FACE Trialists, et al; A randomized controlled trial of endovascular aneurysm repair versus open surgery for abdominal aortic aneurysms in low- to moderate-risk patients. J Vasc Surg 2011;53(05):1167-1173.e1

9 Li B, Khan S, Salata K, et al. A systematic review and meta-analysis of the long-term outcomes of endovascular versus open repair of abdominal aortic aneurysm. J Vasc Surg 2019;70(03):954-969. e30

10 Lederle FA, Freischlag JA, Kyriakides TCOpen Versus Endovascular Repair (OVER) Veterans Affairs Cooperative Study Group, et al; Outcomes following endovascular vs open repair of abdominal aortic aneurysm: a randomized trial. JAMA 2009;302(14): $1535-1542$

11 Rutherford RB. Randomized EVAR trials and advent of level i evidence: a paradigm shift in management of large abdominal aortic aneurysms? Semin Vasc Surg 2006;19(02):69-74

12 Schanzer A, Greenberg RK, Hevelone N, et al. Predictors of abdominal aortic aneurysm sac enlargement after endovascular repair. Circulation 2011;123(24):2848-2855

13 Kent KC. Clinical practice. Abdominal aortic aneurysms. N Engl J Med 2014;371(22):2101-2108

14 Desgranges P, Kobeiter H, Katsahian SECAR Investigators, et al; Editor's choice - ECAR (Endovasculaire ou Chirurgie dans les Anévrysmes aorto-iliaques Rompus): a French randomized controlled trial of endovascular versus open surgical repair of ruptured aorto-iliac aneurysms. Eur J Vasc Endovasc Surg 2015;50 (03):303-310

15 Reimerink JJ, Hoornweg LL, Vahl ACAmsterdam Acute Aneurysm Trial Collaborators, et al; Endovascular repair versus open repair of ruptured abdominal aortic aneurysms: a multicenter randomized controlled trial. Ann Surg 2013;258(02):248-256

16 IMPROVE Trial Investigators. Endovascular strategy or open repair for ruptured abdominal aortic aneurysm: one-year outcomes from the IMPROVE randomized trial. Eur Heart J 2015;36(31): 2061-2069

17 Sweeting MJ, Balm R, Desgranges P, Ulug P, Powell JTRuptured Aneurysm Trialists. Individual-patient meta-analysis of three randomized trials comparing endovascular versus open repair for ruptured abdominal aortic aneurysm. Br J Surg 2015;102(10): 1229-1239

18 Wang LJ, Locham S, Al-Nouri O, Eagleton MJ, Clouse WD, Malas MB. Endovascular repair of ruptured abdominal aortic aneurysm is superior to open repair: Propensity-matched analysis in the Vascular Quality Initiative. J Vasc Surg 2020;72(02):498-507

19 Edwards ST, Schermerhorn ML, O'Malley AJ, et al. Comparative effectiveness of endovascular versus open repair of ruptured abdominal aortic aneurysm in the Medicare population. J Vasc Surg 2014;59(03):575-582

20 Gupta AK, Dakour-Aridi H, Locham S, Nejim B, Veith FJ, Malas MB. Real-world evidence of superiority of endovascular repair in treating ruptured abdominal aortic aneurysm. J Vasc Surg 2018;68(01):74-81

21 Kontopodis N, Tavlas E, Ioannou CV, Giannoukas AD, Geroulakos G, Antoniou GA. Systematic review and meta-analysis of outcomes of open and endovascular repair of ruptured abdominal aortic aneurysm in patients with hostile vs. friendly aortic anatomy. Eur J Vasc Endovasc Surg 2020;59(05):717-728

22 Deery SE, Ergul EA, Schermerhorn MLVascular Study Group of New England, et al; Aneurysm sac expansion is independently associated with late mortality in patients treated with endovascular aneurysm repair. J Vasc Surg 2018;67(01):157-164

23 Antoniou GA, Georgiadis GS, Antoniou SA, et al. Late rupture of abdominal aortic aneurysm after previous endovascular repair: a systematic review and meta-analysis. J Endovasc Ther 2015;22 (05):734-744

24 Herman CR, Charbonneau P, Hongku K, et al. Any nonadherence to instructions for use predicts graft-related adverse events in patients undergoing elective endovascular aneurysm repair. J Vasc Surg 2018;67(01):126-133

25 Kouvelos GN, Oikonomou K, Antoniou GA, Verhoeven ELG, Katsargyris A. A systematic review of proximal neck dilatation after endovascular repair for abdominal aortic aneurysm. J Endovasc Ther 2017;24(01):59-67

26 Oliveira NFG, Gonçalves FB, Hoeks SE, et al. Long-term outcomes of standard endovascular aneurysm repair in patients with severe neck angulation. J Vasc Surg 2018;68(06):1725-1735

27 Thompson JE. Early history of aortic surgery. J Vasc Surg 1998;28 (04):746-752

28 Cooley DA. A brief history of aortic aneurysm surgery. Aorta (Stamford) 2013;1(01):1-3

29 Oransky I. Michael E DeBakey. Lancet 2008;372(9638):530

30 Fairman AS, Chin AL, Jackson BM, et al. The evolution of open abdominal aortic aneurysm repair at a tertiary care center. J Vasc Surg 2020. Doi: 10.1016/j.jvs.2019.12.039 (epub ahead of print)

31 Markar SR, Walsh SR, Griffin K, Khandanpour N, Tang TY, Boyle JR. Assessment of a multifactorial risk index for predicting postoperative pneumonia after open abdominal aortic aneurysm repair. Vascular 2009;17(01):36-39

32 Patel VI, Lancaster RT, Conrad MF, et al. Comparable mortality with open repair of complex and infrarenal aortic aneurysm. J Vasc Surg 2011;54(04):952-959

33 Varkevisser RRB, de Guerre LEMV, Swerdlow NJ, et al. The impact of proximal clamp location on peri-operative outcomes following open surgical repair of juxtarenal abdominal aortic aneurysms. Eur J Vasc Endovasc Surg 2020;59(03):411-418

34 Sarac TP, Clair DG, Hertzer NR, et al. Contemporary results of juxtarenal aneurysm repair. J Vasc Surg 2002;36(06):1104-1111 
35 Oderich GS, Greenberg RK, Farber MZenith Fenestrated Study Investigators, et al; Results of the United States multicenter prospective study evaluating the Zenith fenestrated endovascular graft for treatment of juxtarenal abdominal aortic aneurysms. J Vasc Surg 2014;60(06):1420-8.e1, 5

36 Etkin Y, Baig A, Foley PJ, et al. Management of difficult access during endovascular aneurysm repair. Ann Vasc Surg 2017; 44:77-82

37 Cury M, Zeidan F, Lobato AC. Aortic disease in the young: genetic aneurysm syndromes, connective tissue disorders, and familial aortic aneurysms and dissections. Int J Vasc Med 2013; 2013:267215

38 Kouchoukos NT. Endovascular surgery in Marfan syndrome: CON. Ann Cardiothorac Surg 2017;6(06):677-681

39 Waterman AL, Feezor RJ, Lee WA, et al. Endovascular treatment of acute and chronic aortic pathology in patients with Marfan syndrome. J Vasc Surg 2012;55(05):1234-1240, 1240-1241

40 Nakashima Y, Kurozumi T, Sueishi K, Tanaka K. Dissecting aneurysm: a clinicopathologic and histopathologic study of 111 autopsied cases. Hum Pathol 1990;21(03):291-296

41 Lombardi JV, Cambria RP, Nienaber CASTABLE Investigators, et al; Prospective multicenter clinical trial (STABLE) on the endovascular treatment of complicated type B aortic dissection using a composite device design. J Vasc Surg 2012;55(03):629-640.e2

42 Brunkwall J, Kasprzak P, Verhoeven EADSORB Trialists, et al; Endovascular repair of acute uncomplicated aortic type B dissec- tion promotes aortic remodelling: 1 year results of the ADSORB trial. Eur J Vasc Endovasc Surg 2014;48(03):285-291

43 Schwartz SI, Durham C, Clouse WD, et al. Predictors of late aortic intervention in patients with medically treated type B aortic dissection. J Vasc Surg 2018;67(01):78-84

44 Johnston WF, Upchurch GR, Tracci MC, Cherry KJ, Ailawadi G, Kern JA. Staged hybrid approach using proximal thoracic endovascular aneurysm repair and distal open repair for the treatment of extensive thoracoabdominal aortic aneurysms. J Vasc Surg 2012;56(06):1495-1502

45 Brinster CJ, Fairman RM, Woo EY, Wang GJ, Carpenter JP, Jackson BM. Late open conversion and explantation of abdominal aortic stent grafts. J Vasc Surg 2011;54(01):42-46

46 Scali ST, Mcnally MM, Feezor RJ, et al. Elective endovascular aortic repair conversion for type Ia endoleak is not associated with increased morbidity or mortality compared with primary juxtarenal aneurysm repair. J Vasc Surg 2014;60(02):286-294

47 Jenkins M. EVAR NICE AAA Guidance: Where We Are Now and How We Got Here. Endovascular Today 2020;19(03. Available at: https://evtoday.com/articles/2020-mar/nice-aaa-guidancewhere-we-are-now-and-how-we-got-here. Accessed May 29, 2020

48 Abdominal aortic aneurysm: diagnosis and management. London: National Institute for Health and Care Excellence (UK); 2020. Available at: https://www.nice.org.uk/guidance/gid-cgwave0769/. Accessed May 29, 2020 